\title{
Extraction versus non-extraction orthodontic treatment: Soft tissue profile changes in borderline class I patients
}

\section{Ekstrakcyjne a nieekstrakcyjne leczenie ortodontyczne - zmiany profilu tkanek miękkich u pacjentów z wątpliwą klasą I}

\author{
Sepideh Soheilifar ${ }^{1, A, F}$, Sanaz Soheilifar ${ }^{2, B}$, Hooman Ataei ${ }^{2, B}$, Vahid Mollabashi ${ }^{1, B}$, Payam Amini ${ }^{3, C}$, Anahita Bakhshaei ${ }^{4, D}$, Navid Naghdi ${ }^{, E}$ \\ 1 Department of Orthodontics, Dental Research Center, Hamadan University of Medical Sciences, Iran \\ ${ }^{2}$ Private practice, Hamadan, Iran \\ ${ }^{3}$ Department of Biostatistics and Epidemiology, School of Public Health, Ahvaz Jundishapur University of Medical Sciences, Iran \\ ${ }^{4}$ Department of Orthodontics, Faculty of Dentistry, Hamadan University of Medical Sciences, Iran \\ ${ }^{5}$ Department of Oral and Maxillofacial Surgery, Implant Research Center, Hamadan University of Medical Sciences, Iran \\ A - research concept and design; $\mathrm{B}$ - collection and/or assembly of data; $\mathrm{C}$ - data analysis and interpretation; \\ $D$ - writing the article; $E$ - critical revision of the article; $F$ - final approval of the article
}

Address for correspondence

Anahita Bakhshaei

E-mail:ana.bakhshaei@gmail.com

Funding sources

None declared

Conflict of interest

None declared

Received on November 25, 2019

Reviewed on December 23, 2019

Accepted on March 16, 2020

Published online on September 30, 2020

Cite as

Soheilifar S, Soheilifar S, Ataei H, et al. Extraction versus non-extraction orthodontic treatment: Soft tissue profile changes in borderline class I patients. Dent Med Probl. 2020;57(3):275-283. doi:10.17219/dmp/119102

D0I

$10.17219 / \mathrm{dmp} / 119102$

Copyright

๑ 2020 by Wroclaw Medical University

This is an article distributed under the terms of the

Creative Commons Attribution 3.0 Unported License (CC BY 3.0)

(https://creativecommons.org/licenses/by/3.0/).

\begin{abstract}
Background. The decision regarding the selection of extraction or non-extraction orthodontic treatment is a common challenge in orthodontic treatment planning.

Objectives. The objective of this study was to compare the effects of extraction and non-extraction orthodontic treatment on the soft tissue profile of borderline class I patients.

Material and methods. In this retrospective study, 70 patients were selected from among those referred to the Department of Orthodontics of the Faculty of Dentistry at Hamadan University of Medical Sciences in Iran. The inclusion criteria were skeletal class I, 4-10 mm of space deficiency, and the possibility of applying both extraction and non-extraction orthodontic treatment. All patients underwent fixed orthodontic treatment with the use of 0.022 -inch-slot edgewise brackets. The patients were divided into 2 groups $(n=35)$ according to the 4-premolar extraction or non-extraction treatment plan. The 2 groups were compared by means of the cephalometric analysis.

Results. There were 11 males and 24 females at a mean age of 17.46 years in the non-extraction group, and 9 males and 26 females at a mean age of 18.46 years in the extraction group. The upper and lower incisors as well as the lower lip moved forward in the non-extraction group (lower lip to E-plane $=0.87 \pm 1.39 \mathrm{~mm}, \mathrm{U1}-\mathrm{SN}=2.83 \pm 8.03^{\circ}, \mathrm{IMPA}=4.64 \pm 5.47^{\circ}$. The incisors and the lips moved backward in the extraction group (upper lip to $\mathrm{E}$-plane $=-1.42 \pm 2.08 \mathrm{~mm}$, lower lip to $\mathrm{E}$-plane $\left.=-1.56 \pm 1.97 \mathrm{~mm}, \mathrm{U1}-\mathrm{SN}=-7.63 \pm 9.02^{\circ}, \mathrm{IMPA}=-7.05 \pm 6.79^{\circ}\right)$. The differences were statistically significant $(p<0.05)$. Mentolabial sulcus became more pronounced in the non-extraction group $(1.92 \pm 2.73 \mathrm{~mm} ; p<0.001)$ and shallower in the extraction group $(-1.90 \pm 4.2 \mathrm{~mm} ; p=0.000)$.

Conclusions. Orthodontic treatment can change the soft tissue appearance of the lower third of the face. The lips and the incisors moved forward in the non-extraction group and backward in the extraction group.

Key words: tooth extraction, orthodontics, cephalometry, Angle class I malocclusion
\end{abstract}

Słowa kluczowe: ekstrakcja zęba, ortodoncja, cefalometria, wada zgryzu klasy I wg Angle'a 


\section{Introduction}

A large number of people seek orthodontic treatment to improve their dentofacial appearance. ${ }^{1}$ In general, the purpose of orthodontic treatment is to improve dentofacial appearance, oral function and the stability of the dental arches. Choosing an appropriate treatment plan helps to maintain a balance between the 3 above-mentioned parameters. $^{2-4}$

The decision regarding the creation of space is challenging in orthodontic treatment planning. In patients with the tooth size-arch size discrepancy, it is necessary to provide sufficient space for the teeth during orthodontic treatment. The conventional methods for creating the space include the distal movement of the posterior teeth, changes in the width and shape of the arch, the interproximal reduction of the teeth, the protrusion of the incisors, and tooth extraction. Tooth extraction may be required to achieve a coordinated dental arch, compensate moderate to severe dental protrusion or crowding, and camouflage mild to moderate jaw discrepancies. ${ }^{1}$ The decision regarding tooth extraction depends on different factors, such as esthetic considerations, the stability of the teeth, and the preferences of patients or orthodontists. ${ }^{5}$

Non-extraction orthodontic treatment is indicated in patients with space excess or dental retroclination. In contrast, in the case of space shortage or dental proclination, the only treatment option is tooth extraction. The treatment plan in such orthodontic patients is relatively straightforward. In addition, post-treatment outcomes in such patients are highly predictable. However, there are some patients for whom both extraction and nonextraction orthodontic treatment can be considered. In such patients, the choice of treatment depends on several factors, and the treatment plan may vary, depending on the patient's soft and hard tissue components, and the preferences of orthodontists and patients. The choice of the treatment plan in these patients is considered to be borderline. No consensus has been reached regarding the effects of tooth extraction on the vertical dimension, facial profile changes, jaw position, temporomandibular joint health, and the periodontal status after treatment. ${ }^{6,7}$

The perspectives of patients and orthodontists regarding an ideal soft tissue profile have been continuously changing over the past decades. ${ }^{8}$ Tooth extraction can change the soft tissue profile. However, there is controversy regarding the long-term effects of tooth extraction. ${ }^{9}$ Although some studies have been conducted on this topic, no study could reach a definite conclusion regarding the effect of treatment on the appearance of the lower third of the face. ${ }^{8,10-12}$ A limited number of available studies on this topic include borderline patients. The results may be overestimated or underestimated, depending on whether the chosen patients had space shortage or excess, or dental protrusion.

The primary objective of this study was to evaluate and compare the effects of extraction and non-extraction orthodontic treatment on the soft tissue profile of borderline class I patients.

\section{Material and methods}

In this retrospective study, cephalometric changes that occurred during the orthodontic treatment of the patients treated with extraction and non-extraction methods were evaluated. The patients were randomly selected from among those reporting to the Department of Orthodontics of the Faculty of Dentistry at Hamadan University of Medical Sciences in Iran.

The inclusion criteria were as follows: age between 18 and 30 years, skeletal class I relationship $\left(1^{\circ}<\mathrm{ANB}<5^{\circ}\right)$, space deficiency of $4-10 \mathrm{~mm}$, and the borderline treatment plan - the possibility of both extraction and nonextraction treatment.

The exclusion criteria were the following: patients with craniofacial deformities or syndromes, large discrepancies in the tooth size, a history of unsuccessful orthodontic or orthognathic treatment, a history of facial surgeries, an intake of medications that would affect the soft tissue volume.

In order to choose borderline patients, 6 factors were assessed according to Paquette et al. ${ }^{13}$ : (1) the magnitude of space deficiency in the maxillary arch; (2) the magnitude of space deficiency in the mandibular arch; (3) maxillary incisor protrusion (U1-SN); (4) mandibular incisor protrusion (L1-NB); (5) the irregularity index; and (6) facial convexity (Z-angle).

Afterward, 3 orthodontists were asked to design the treatment plan according to the 6 above-mentioned factors. The patients were categorized in the borderline range in case of disagreement between the orthodontists regarding the selection of the extraction or non-extraction orthodontic treatment plan.

The sample size was calculated to be 34 in each group according to a previous study, ${ }^{1}$ assuming a power of 0.85 , type I error of 0.05 , standard deviation $(S D)$ of 1.2 , and with the aim of finding a 0.5 -millimeter true difference between the groups, using Power and Sample Size Calculator v. 3.1.2 (https://ps-power-and-sample-size-calculation.software.informer.com/3.1/).

The primary (baseline) and final cephalograms, casts and photographs of the patients were examined for space deficiency, and the soft tissue profile analysis was performed for case selection. All patients had been treated with 0.022 -inch-slot edgewise brackets, and had complete pre-treatment and post-treatment diagnostic records.

The eligible patients were categorized as follows:

- the non-extraction group included patients who underwent non-extraction orthodontic treatment (except third molar extraction);

- the extraction group included patients who were treated with the extraction of the 4 first or second premolars. 
The pre-treatment and post-treatment cephalograms were analyzed using the Dolphin Imaging software v. 11.8 (Patterson Dental Supply, Inc., St. Paul, USA). Thirty-seven landmarks were assessed (23 hard tissue and 14 soft tissue landmarks) (Fig. 1). Linear and angular measurements were made to evaluate the soft and hard tissue profiles of the patients (Table 1).

In order to assess the intra-class correlation coefficient, 11 cephalograms were traced by another calibrated researcher.

The data was analyzed using PASW Statistics for Windows v. 18 (SPSS, Inc., Chicago, USA). The paired $t$ test was used to analyze changes in each treatment group, and the independent $t$ test was utilized to compare the 2 kinds of treatment in the 2 groups. Pearson's correlation coefficient was used to assess the correlation of hard tissue and soft tissue changes. A $p$-value of $<0.05$ was considered statistically significant.

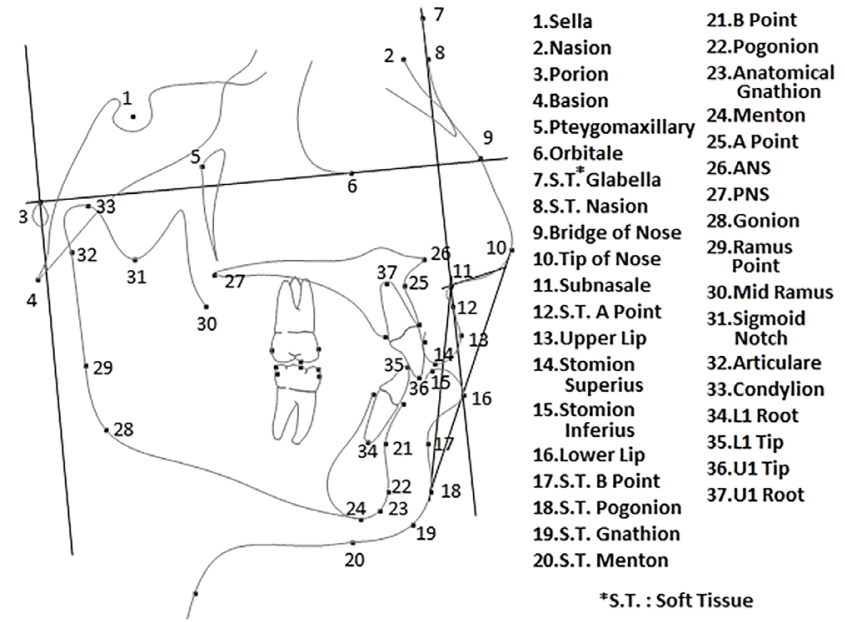

Fig. 1. Hard tissue and soft tissue cephalometric landmarks used for cephalometric tracing

Table 1. Linear and angular cephalometric measurements and their definitions

\begin{tabular}{|c|c|c|}
\hline No. & Variable & Definition \\
\hline 1 & $\begin{array}{l}\text { nasolabial angle } \\
\quad(\text { Col-Sn-UL) }\end{array}$ & angle between the line tangent to the columella and the line tangent to the upper lip \\
\hline 2 & E-plane & line passing through the tip of the nose and soft tissue pogonion \\
\hline 3 & upper lip to E-plane & distance of the most anterior point of the upper lip to E-plane \\
\hline 4 & lower lip to E-plane & distance of the most anterior point of the lower lip to E-plane \\
\hline 5 & upper lip thickness & horizontal distance between the most anterior point of the upper lip and the labial surface of the upper central incisors \\
\hline 6 & lower lip thickness & horizontal distance between the most anterior point of the lower lip and the labial surface of the lower central incisors \\
\hline 7 & upper lip length & the shortest distance between subnasale and the most inferior point of the upper lip vermilion \\
\hline 8 & lower lip length & the shortest distance between soft tissue menton and the most superior point of the lower lip vermilion \\
\hline 9 & mentolabial sulcus & $\begin{array}{l}\text { the shortest distance between the most posterior point in the lower lip contour (soft tissue B point) } \\
\text { and the line passing through soft tissue pogonion and the most anterior point of the lower lip }\end{array}$ \\
\hline 10 & Wits appraisal & distance between the points resulting from drawing perpendicular lines from A and B points to the functional occlusal plane \\
\hline 11 & $\begin{array}{l}\text { post.-ant. face height } \\
\qquad(\mathrm{S}-\mathrm{Go} / \mathrm{N}-\mathrm{Me})\end{array}$ & proportional ratio between the posterior facial height ( $\mathrm{S}$ to gonion) and the anterior facial height ( $\mathrm{N}$ to menton) \\
\hline 12 & $\begin{array}{c}\text { UFH / TFH } \\
(\mathrm{N}-\mathrm{ANS} / \mathrm{N}-\mathrm{Me})\end{array}$ & $\begin{array}{l}\text { proportional ratio between the upper anterior facial height ( } N \text { to ANS point) and the total anterior facial height } \\
\text { ( } N \text { to menton) }\end{array}$ \\
\hline 13 & $\begin{array}{l}\text { posterior facial height } \\
\qquad \text { (S-Go) }\end{array}$ & distance between $\mathrm{S}$ and gonion \\
\hline 14 & $\begin{array}{l}\text { anterior facial height } \\
\qquad(\mathrm{N}-\mathrm{Me})\end{array}$ & distance between $\mathrm{N}$ and menton \\
\hline 15 & $\begin{array}{l}\text { upper face height } \\
\text { (N-ANS) }\end{array}$ & distance between $\mathrm{N}$ and ANS point \\
\hline 16 & Pog-NB & distance between pogonion and the line passing through $\mathrm{N}$ and $\mathrm{B}$ points \\
\hline 17 & SN-GoMe & angle between SN line and the mandibular plane (gonion to menton line) \\
\hline 18 & U1-SN & angle between the long axis of the most prominent upper incisors and SN line \\
\hline 19 & interincisal angle & angle between the long axis of the upper and lower incisors \\
\hline 20 & $\begin{array}{l}\text { IMPA } \\
(\mathrm{L} 1-\mathrm{MP})\end{array}$ & $\begin{array}{l}\text { angle between the long axis of the most prominent lower incisors and the mandibular plane } \\
\qquad \text { (gonion to menton line) }\end{array}$ \\
\hline 21 & $\mathrm{FH}-\mathrm{SN}$ & angle between Frankfort and SN lines \\
\hline 22 & SNA & angle between S, N and A points \\
\hline 23 & SNB & angle between $\mathrm{S}, \mathrm{N}$ and $\mathrm{B}$ points \\
\hline 24 & ANB & angle between $\mathrm{A}, \mathrm{N}$ and $\mathrm{B}$ points \\
\hline 25 & interlabial gap & tical distance between the most inferior point of the upper lip vermilion and the most superior point of the lower lip vermilion \\
\hline
\end{tabular}




\section{Results}

This study was performed on 70 patients, including 35 patients in the extraction group (11 males and 24 females) and 35 patients in the non-extraction group (9 males and 26 females). The mean age of the patients at the onset of treatment was $17.45 \pm 4.66$ years and $18.46 \pm 4.94$ years in the non-extraction and extraction groups, respectively. The mean intra-class correlation coefficient was 0.79 .

Tables 2 and 3 show the pre-treatment and posttreatment values of cephalometric variables in the non-extraction and extraction groups, respectively.

In the non-extraction group, changes in lower lip to E-plane $(p \leq 0.001)$, mentolabial sulcus $(p \leq 0.001)$, SN-GoMe $(p \leq 0.035)$, U1-SN $(p \leq 0.044)$, interincisal angle $(p \leq 0.001)$, and IMPA $(p \leq 0.001)$ were all statistically significant.

In the extraction group, nasolabial angle $(p \leq 0.048)$, upper lip to E-plane $(p \leq 0.001)$, lower lip to E-plane $(p \leq 0.001)$, mentolabial sulcus $(p \leq 0.012)$, Pog-NB $(p \leq 0.047), \mathrm{U} 1-\mathrm{SN}(p \leq 0.001)$, interincisal angle $(p \leq 0.001)$, and IMPA $(p \leq 0.001)$ changed significantly after treatment.

Table 4 compares the changes between the extraction and non-extraction treatment groups.

The comparison of the 2 groups revealed significant differences in the upper lip to E-plane, lower lip to E-plane, mentolabial sulcus, U1-SN, interincisal angle, and IMPA (Table 4).

Significant differences were noted between the extraction and non-extraction treatment groups regarding the change in the position of the upper and lower incisors and the lips, all moving backward in the extraction group. The upper and lower incisors and the lower lip moved forward in the non-extraction treatment group. Mentolabial sulcus became shallower in the non-extraction group and more pronounced in the extraction group.

Tables 5 and 6 show the results of Pearson's correlation test regarding the correlation between skeletal/dental changes and soft tissue changes.

As shown in Tables 5 and 6, the posterior and anterior facial height had a significant correlation with the upper and lower lip length and the mentolabial sulcus depth in both groups and with the upper lip thickness in the extraction group. The parameter Pog-NB had an inverse correlation with lower lip to E-plane in the extraction group, while there was a positive correlation between this parameter and the lower lip length, and U1-SN had a moderately positive correlation with the upper lip thickness and the mentolabial sulcus depth in the non-extraction group. The parameter IMPA positively correlated with the upper lip thickness and the mentolabial sulcus depth in the extraction group.
Table 2. Pre-treatment and post-treatment values of the variables in the non-extraction group

\begin{tabular}{|c|c|c|c|c|}
\hline No. & Variable & $\begin{array}{l}\text { Before } \\
\text { treatment }\end{array}$ & $\begin{array}{l}\text { After } \\
\text { treatment }\end{array}$ & $p$-value \\
\hline 1 & $\begin{array}{c}\text { nasolabial angle } \\
\text { (Col-Sn-UL) } \\
{\left[{ }^{\circ}\right]}\end{array}$ & $107.81 \pm 10.38$ & $107.02 \pm 9.84$ & 0.676 \\
\hline 2 & $\begin{array}{l}\text { upper lip to E-plane } \\
\text { [mm] }\end{array}$ & $-3.20 \pm 2.16$ & $-3.20 \pm 2.01$ & 0.992 \\
\hline 3 & $\begin{array}{l}\text { lower lip to E-plane } \\
{[\text { [mm] }}\end{array}$ & $-0.37 \pm 2.12$ & $0.50 \pm 2.33$ & $0.001^{*}$ \\
\hline 4 & $\begin{array}{l}\text { upper lip thickness } \\
{[\mathrm{mm}]}\end{array}$ & $4.53 \pm 1.07$ & $4.64 \pm 1.19$ & 0.645 \\
\hline 5 & $\begin{array}{l}\text { lower lip thickness } \\
{[\mathrm{mm}]}\end{array}$ & $4.55 \pm 1.12$ & $4.76 \pm 1.17$ & 0.574 \\
\hline 6 & $\begin{array}{l}\text { upper lip length } \\
\text { [mm] }\end{array}$ & $20.19 \pm 2.50$ & $19.84 \pm 3.30$ & 0.439 \\
\hline 7 & $\begin{array}{l}\text { lower lip length } \\
{[\mathrm{mm}]}\end{array}$ & $17.05 \pm 3.14$ & $17.09 \pm 2.77$ & 0.943 \\
\hline 8 & $\begin{array}{l}\text { mentolabial sulcus } \\
{[\mathrm{mm}]}\end{array}$ & $7.52 \pm 3.87$ & $9.44 \pm 4.43$ & $0.001^{*}$ \\
\hline 9 & $\begin{array}{l}\text { Wits appraisal } \\
\qquad[\mathrm{mm}]\end{array}$ & $2.18 \pm 3.41$ & $2.00 \pm 2.53$ & 0.129 \\
\hline 10 & $\begin{array}{l}\text { post.-ant. face height } \\
\text { (S-Go/N-Me) }\end{array}$ & $63.73 \pm 3.37$ & $63.86 \pm 3.64$ & 0.757 \\
\hline 11 & $\begin{array}{c}\text { UFH / TFH } \\
\text { (N-ANS / N-Me) }\end{array}$ & $44.28 \pm 1.98$ & $44.50 \pm 2.11$ & 0.401 \\
\hline 12 & $\begin{array}{l}\text { posterior facial height } \\
\qquad \text { (S-Go) } \\
{[\mathrm{mm}]}\end{array}$ & $70.11 \pm 8.53$ & $70.54 \pm 10.39$ & 0.776 \\
\hline 13 & $\begin{array}{l}\text { anterior facial height } \\
\text { (N-Me) } \\
{[\mathrm{mm}]}\end{array}$ & $121.62 \pm 12.71$ & $123.17 \pm 15.80$ & 0.533 \\
\hline 14 & $\begin{array}{l}\text { upper face height } \\
\text { (N-ANS) } \\
{[\mathrm{mm}]}\end{array}$ & $47.76 \pm 5.34$ & $48.59 \pm 6.13$ & 0.427 \\
\hline 15 & $\begin{array}{l}\text { Pog-NB } \\
{[\mathrm{mm}]}\end{array}$ & $1.48 \pm 1.29$ & $1.46 \pm 1.46$ & 0.929 \\
\hline 16 & $\begin{array}{c}\text { SN-GoMe } \\
{\left[{ }^{\circ}\right]}\end{array}$ & $34.06 \pm 4.50$ & $35.21 \pm 4.74$ & $0.035^{*}$ \\
\hline 17 & $\begin{array}{c}\text { U1-SN } \\
{\left[{ }^{\circ}\right]}\end{array}$ & $102.91 \pm 7.17$ & $105.74 \pm 6.85$ & $0.044^{*}$ \\
\hline 18 & $\begin{array}{c}\text { interincisal angle } \\
{\left[{ }^{\circ}\right]}\end{array}$ & $125.15 \pm 10.37$ & $116.55 \pm 9.44$ & $0.001^{*}$ \\
\hline 19 & $\begin{array}{c}\text { IMPA } \\
(\mathrm{L} 1-\mathrm{MP}) \\
{\left[{ }^{\circ}\right]}\end{array}$ & $95.08 \pm 6.00$ & $99.72 \pm 6.87$ & $0.001^{*}$ \\
\hline 20 & $\begin{array}{c}\mathrm{FH}-\mathrm{SN} \\
{\left[{ }^{\circ}\right]}\end{array}$ & $7.36 \pm 3.02$ & $6.96 \pm 3.18$ & 0.467 \\
\hline 21 & $\begin{array}{c}\text { SNA } \\
{\left[{ }^{\circ}\right]}\end{array}$ & $82.31 \pm 4.59$ & $81.68 \pm 4.38$ & 0.265 \\
\hline 22 & $\begin{array}{c}\text { SNB } \\
{\left[{ }^{\circ}\right]}\end{array}$ & $76.84 \pm 3.04$ & $77.00 \pm 3.11$ & 0.694 \\
\hline 23 & $\begin{array}{c}\text { ANB } \\
\left.{ }^{\circ}\right]\end{array}$ & $4.47 \pm 2.54$ & $4.69 \pm 2.35$ & 0.115 \\
\hline 24 & $\begin{array}{l}\text { interlabial gap } \\
{[\mathrm{mm}]}\end{array}$ & $2.09 \pm 1.53$ & $1.95 \pm 0.67$ & 0.594 \\
\hline
\end{tabular}

Data presented as mean $(M) \pm$ standard deviation (SD). * statistical significance. 
Table 3. Pre-treatment and post-treatment values of the variables in the extraction group

\begin{tabular}{|c|c|c|c|c|}
\hline No. & Variable & $\begin{array}{l}\text { Before } \\
\text { treatment }\end{array}$ & $\begin{array}{c}\text { After } \\
\text { treatment }\end{array}$ & $p$-value \\
\hline 1 & $\begin{array}{c}\text { nasolabial angle } \\
\text { (Col-Sn-UL) } \\
{\left[{ }^{\circ}\right]}\end{array}$ & $107.12 \pm 13.75$ & $111.15 \pm 11.28$ & $0.048^{*}$ \\
\hline 2 & $\begin{array}{l}\text { upper lip to E-plane } \\
\text { [mm] }\end{array}$ & $-2.27 \pm 2.06$ & $-3.69 \pm 2.05$ & $0.001^{*}$ \\
\hline 3 & $\begin{array}{l}\text { lower lip to E-plane } \\
{[\mathrm{mm}]}\end{array}$ & $0.80 \pm 2.25$ & $-0.76 \pm 2.08$ & $0.001^{*}$ \\
\hline 4 & $\begin{array}{l}\text { upper lip thickness } \\
{[\mathrm{mm}]}\end{array}$ & $4.40 \pm 1.15$ & $4.55 \pm 1.10$ & 0.674 \\
\hline 5 & $\begin{array}{l}\text { lower lip thickness } \\
{[\mathrm{mm}]}\end{array}$ & $4.43 \pm 1.63$ & $4.62 \pm 1.53$ & 0.703 \\
\hline 6 & $\begin{array}{l}\text { upper lip length } \\
{[\mathrm{mm}]}\end{array}$ & $19.84 \pm 3.52$ & $20.00 \pm 3.25$ & 0.806 \\
\hline 7 & $\begin{array}{c}\text { lower lip length } \\
{[\mathrm{mm}]}\end{array}$ & $16.66 \pm 3.22$ & $17.29 \pm 2.68$ & 0.312 \\
\hline 8 & $\begin{array}{c}\text { mentolabial sulcus } \\
{[\mathrm{mm}]}\end{array}$ & $9.04 \pm 5.42$ & $7.14 \pm 3.56$ & $0.012^{*}$ \\
\hline 9 & $\begin{array}{l}\text { Wits appraisal } \\
\text { [mm] }\end{array}$ & $1.63 \pm 3.52$ & $1.19 \pm 3.32$ & 0.434 \\
\hline 10 & $\begin{array}{l}\text { post.-ant. face height } \\
\text { (S-Go / N-Me) }\end{array}$ & $62.72 \pm 3.48$ & $63.40 \pm 3.64$ & 0.193 \\
\hline 11 & $\begin{array}{c}\text { UFH / TFH } \\
\text { (N-ANS / N-Me) }\end{array}$ & $44.00 \pm 2.08$ & $43.94 \pm 1.96$ & 0.806 \\
\hline 12 & $\begin{array}{l}\text { posterior facial height } \\
\text { (S-Go) } \\
{[\mathrm{mm}]}\end{array}$ & $69.27 \pm 10.99$ & $71.80 \pm 9.28$ & 0.245 \\
\hline 13 & $\begin{array}{l}\text { anterior facial height } \\
(\mathrm{N}-\mathrm{Me}) \\
{[\mathrm{mm}]}\end{array}$ & $121.26 \pm 17.78$ & $125.98 \pm 14.76$ & 0.212 \\
\hline 14 & $\begin{array}{l}\text { upper face height } \\
\text { (N-ANS) } \\
{[\mathrm{mm}]}\end{array}$ & $47.25 \pm 6.70$ & $49.09 \pm 5.61$ & 0.218 \\
\hline 15 & $\begin{array}{l}\text { Pog-NB } \\
{[\mathrm{mm}]}\end{array}$ & $0.86 \pm 1.21$ & $1.24 \pm 1.21$ & $0.047^{*}$ \\
\hline 16 & $\begin{array}{c}\text { SN-GoMe } \\
{\left[^{\circ}\right]}\end{array}$ & $35.05 \pm 5.15$ & $35.96 \pm 5.54$ & 0.057 \\
\hline 17 & $\begin{array}{c}\text { U1-SN } \\
{\left[^{\circ}\right]}\end{array}$ & $105.96 \pm 7.59$ & $98.33 \pm 9.04$ & $0.001^{*}$ \\
\hline 18 & $\begin{array}{c}\text { interincisal angle } \\
{\left[{ }^{\circ}\right]}\end{array}$ & $119.76 \pm 11.43$ & $133.39 \pm 8.29$ & $0.001^{*}$ \\
\hline 19 & $\begin{array}{c}\text { IMPA } \\
(\mathrm{L} 1-\mathrm{MP}) \\
{\left[^{\circ}\right]}\end{array}$ & $96.22 \pm 6.90$ & $89.17 \pm 7.26$ & $0.001^{*}$ \\
\hline 20 & $\begin{array}{c}\mathrm{FH}-\mathrm{SN} \\
{\left[{ }^{\circ}\right]}\end{array}$ & $7.34 \pm 3.35$ & $7.06 \pm 3.41$ & 0.647 \\
\hline 21 & $\begin{array}{c}\text { SNA } \\
{\left[^{\circ}\right]}\end{array}$ & $81.32 \pm 3.53$ & $81.21 \pm 3.87$ & 0.798 \\
\hline 22 & $\begin{array}{l}\text { SNB } \\
{\left[{ }^{\circ}\right]}\end{array}$ & $76.89 \pm 3.14$ & $76.76 \pm 4.05$ & 0.692 \\
\hline 23 & $\begin{array}{c}\text { ANB } \\
\left.{ }^{\circ}\right]\end{array}$ & $4.43 \pm 2.71$ & $4.46 \pm 2.50$ & 0.947 \\
\hline 24 & $\begin{array}{l}\text { interlabial gap } \\
{[\mathrm{mm}]}\end{array}$ & $2.28 \pm 2.07$ & $2.13 \pm 1.18$ & 0.648 \\
\hline
\end{tabular}

Data presented as mean $(M) \pm$ standard deviation (SD).

* statistical significance.
Table 4. Comparison of the treatment changes between the extraction and non-extraction groups

\begin{tabular}{|c|c|c|c|c|}
\hline Parameter & Group & $M$ & $S D$ & $p$-value \\
\hline $\begin{array}{c}\text { nasolabial angle } \\
\text { (Col-Sn-UL) } \\
\left.{ }^{\circ}\right]\end{array}$ & $\begin{array}{c}\text { non-extraction } \\
\text { extraction }\end{array}$ & $\begin{array}{r}-0.789 \\
4.031\end{array}$ & $\begin{array}{l}11.05049 \\
11.63786\end{array}$ & 0.080 \\
\hline $\begin{array}{l}\text { upper lip to E-plane } \\
\text { [mm] }\end{array}$ & $\begin{array}{c}\text { non-extraction } \\
\text { extraction }\end{array}$ & $\begin{array}{l}-0.003 \\
-1.423\end{array}$ & $\begin{array}{l}1.76993 \\
2.08144\end{array}$ & $0.003^{*}$ \\
\hline $\begin{array}{l}\text { lower lip to E-plane } \\
\text { [mm] }\end{array}$ & $\begin{array}{c}\text { non-extraction } \\
\text { extraction }\end{array}$ & $\begin{array}{r}0.874 \\
-1.569\end{array}$ & $\begin{array}{l}1.39439 \\
1.97153\end{array}$ & $0.001^{*}$ \\
\hline $\begin{array}{l}\text { upper lip thickness } \\
{[\mathrm{mm}]}\end{array}$ & $\begin{array}{c}\text { non-extraction } \\
\text { extraction }\end{array}$ & $\begin{array}{l}0.209 \\
0.204\end{array}$ & $\begin{array}{l}1.28941 \\
1.34009\end{array}$ & 0.439 \\
\hline $\begin{array}{l}\text { lower lip thickness } \\
{[\mathrm{mm}]}\end{array}$ & $\begin{array}{c}\text { non-extraction } \\
\text { extraction }\end{array}$ & $\begin{array}{l}0.274 \\
0.191\end{array}$ & $\begin{array}{l}2.02068 \\
2.61745\end{array}$ & 0.461 \\
\hline $\begin{array}{l}\text { upper lip length } \\
{[\mathrm{mm}]}\end{array}$ & $\begin{array}{c}\text { non-extraction } \\
\text { extraction }\end{array}$ & $\begin{array}{r}-0.351 \\
0.166\end{array}$ & $\begin{array}{l}2.65766 \\
3.96002\end{array}$ & 0.523 \\
\hline $\begin{array}{c}\text { lower lip length } \\
{[\mathrm{mm}]}\end{array}$ & $\begin{array}{c}\text { non-extraction } \\
\text { extraction }\end{array}$ & $\begin{array}{l}0.037 \\
0.631\end{array}$ & $\begin{array}{l}3.03858 \\
3.64206\end{array}$ & 0.461 \\
\hline $\begin{array}{c}\text { mentolabial sulcus } \\
{[\mathrm{mm}]}\end{array}$ & $\begin{array}{c}\text { non-extraction } \\
\text { extraction }\end{array}$ & $\begin{array}{r}1.911 \\
-1.891\end{array}$ & $\begin{array}{l}2.73354 \\
4.20423\end{array}$ & $0.001^{*}$ \\
\hline $\begin{array}{l}\text { Wits appraisal } \\
{[\mathrm{mm}]}\end{array}$ & $\begin{array}{c}\text { non-extraction } \\
\text { extraction }\end{array}$ & $\begin{array}{l}-1.186 \\
-0.443\end{array}$ & $\begin{array}{l}3.07630 \\
3.31044\end{array}$ & 0.334 \\
\hline $\begin{array}{l}\text { post.-ant. face height } \\
\text { (S-Go/ N-Me) }\end{array}$ & $\begin{array}{c}\text { non-extraction } \\
\text { extraction }\end{array}$ & $\begin{array}{l}-0.129 \\
-0.671\end{array}$ & $\begin{array}{l}2.44078 \\
2.98847\end{array}$ & 0.408 \\
\hline $\begin{array}{c}\text { UFH / TFH } \\
\text { (N-ANS / N-Me) }\end{array}$ & $\begin{array}{c}\text { non-extraction } \\
\text { extraction }\end{array}$ & $\begin{array}{r}0.214 \\
-0.054\end{array}$ & $\begin{array}{l}1.49098 \\
1.29985\end{array}$ & 0.425 \\
\hline $\begin{array}{l}\text { posterior facial height } \\
\text { (S-Go) } \\
{[\mathrm{mm}]}\end{array}$ & $\begin{array}{c}\text { non-extraction } \\
\text { extraction }\end{array}$ & $\begin{array}{l}0.431 \\
2.526\end{array}$ & $\begin{array}{r}8.90640 \\
12.63386\end{array}$ & 0.426 \\
\hline $\begin{array}{l}\text { anterior facial height } \\
(\mathrm{N}-\mathrm{Me}) \\
{[\mathrm{mm}]}\end{array}$ & $\begin{array}{c}\text { non-extraction } \\
\text { extraction }\end{array}$ & $\begin{array}{l}1.557 \\
4.723\end{array}$ & $\begin{array}{l}14.63691 \\
21.94631\end{array}$ & 0.480 \\
\hline $\begin{array}{l}\text { upper face height } \\
\text { (N-ANS) } \\
{[\mathrm{mm}]}\end{array}$ & $\begin{array}{c}\text { non-extraction } \\
\text { extraction }\end{array}$ & $\begin{array}{l}0.834 \\
1.843\end{array}$ & $\begin{array}{l}6.13615 \\
8.68671\end{array}$ & 0.577 \\
\hline $\begin{array}{l}\text { Pog-NB } \\
{[\mathrm{mm}]}\end{array}$ & $\begin{array}{c}\text { non-extraction } \\
\text { extraction }\end{array}$ & $\begin{array}{r}-0.014 \\
0.380\end{array}$ & $\begin{array}{l}0.93969 \\
1.09270\end{array}$ & 0.110 \\
\hline $\begin{array}{c}\text { SN-GoMe } \\
{\left[{ }^{\circ}\right]}\end{array}$ & $\begin{array}{c}\text { non-extraction } \\
\text { extraction }\end{array}$ & $\begin{array}{l}1.157 \\
0.911\end{array}$ & $\begin{array}{l}3.11847 \\
2.74084\end{array}$ & 0.727 \\
\hline $\begin{array}{c}\text { U1-SN } \\
{\left[{ }^{\circ}\right]}\end{array}$ & $\begin{array}{c}\text { non-extraction } \\
\text { extraction }\end{array}$ & $\begin{array}{r}2.834 \\
-7.631\end{array}$ & $\begin{array}{l}8.03587 \\
9.02848\end{array}$ & $0.001^{*}$ \\
\hline $\begin{array}{c}\text { interincisal angle } \\
{\left[{ }^{\circ}\right]}\end{array}$ & $\begin{array}{c}\text { non-extraction } \\
\text { extraction }\end{array}$ & $\begin{array}{r}-8.603 \\
13.626\end{array}$ & $\begin{array}{l}11.00036 \\
12.33518\end{array}$ & $0.001^{*}$ \\
\hline $\begin{array}{c}\text { IMPA } \\
(\mathrm{L} 1-\mathrm{MP}) \\
{\left[^{\circ}\right]}\end{array}$ & $\begin{array}{c}\text { non-extraction } \\
\text { extraction }\end{array}$ & $\begin{array}{r}4.637 \\
-7.051\end{array}$ & $\begin{array}{l}5.47379 \\
6.79945\end{array}$ & $0.001^{*}$ \\
\hline $\begin{array}{c}\mathrm{FH}-\mathrm{SN} \\
{\left[{ }^{\circ}\right]}\end{array}$ & $\begin{array}{c}\text { non-extraction } \\
\text { extraction }\end{array}$ & $\begin{array}{l}-0.397 \\
-0.274\end{array}$ & $\begin{array}{l}3.19333 \\
3.50466\end{array}$ & 0.878 \\
\hline $\begin{array}{c}\text { SNA } \\
{\left[{ }^{\circ}\right]}\end{array}$ & $\begin{array}{c}\text { non-extraction } \\
\text { extraction }\end{array}$ & $\begin{array}{l}-0.626 \\
-0.117\end{array}$ & $\begin{array}{l}3.26914 \\
2.68892\end{array}$ & 0.480 \\
\hline $\begin{array}{l}\text { SNB } \\
{\left[{ }^{\circ}\right]}\end{array}$ & $\begin{array}{c}\text { non-extraction } \\
\text { extraction }\end{array}$ & $\begin{array}{r}0.154 \\
-0.131\end{array}$ & $\begin{array}{l}2.30298 \\
1.94766\end{array}$ & 0.577 \\
\hline $\begin{array}{c}\text { ANB } \\
{\left[{ }^{\circ}\right]}\end{array}$ & $\begin{array}{c}\text { non-extraction } \\
\text { extraction }\end{array}$ & $\begin{array}{r}-0.780 \\
0.029\end{array}$ & $\begin{array}{l}1.80274 \\
2.50301\end{array}$ & 0.126 \\
\hline $\begin{array}{c}\text { interlabial gap } \\
{[\mathrm{mm}]}\end{array}$ & $\begin{array}{c}\text { non-extraction } \\
\text { extraction }\end{array}$ & $\begin{array}{l}-0.146 \\
-0.149\end{array}$ & $\begin{array}{l}1.60374 \\
1.90593\end{array}$ & 0.995 \\
\hline
\end{tabular}

M - mean; SD - standard deviation; * statistical significance. 
Table 5. Pearson's correlation between skeletal/dental changes and soft tissue changes in the non-extraction group

\begin{tabular}{|c|c|c|c|c|c|c|c|c|}
\hline $\begin{array}{c}\text { Changes } \\
\text { in parameters }\end{array}$ & $\begin{array}{l}\text { Nasolabial angle } \\
\text { (Col-Sn-UL) }\end{array}$ & $\begin{array}{l}\text { Upper lip to } \\
\text { E-plane }\end{array}$ & $\begin{array}{l}\text { Lower lip to } \\
\text { E-plane }\end{array}$ & $\begin{array}{l}\text { Upper lip } \\
\text { thickness }\end{array}$ & $\begin{array}{l}\text { Lower lip } \\
\text { thickness }\end{array}$ & $\begin{array}{l}\text { Upper lip } \\
\text { length }\end{array}$ & $\begin{array}{l}\text { Lower lip } \\
\text { length }\end{array}$ & $\begin{array}{l}\text { Mentolabial } \\
\text { sulcus }\end{array}$ \\
\hline $\begin{array}{l}\text { Posterior facial height } \\
\text { (S-Go) }\end{array}$ & 0.022 & -0.197 & 0.251 & 0.321 & -0.088 & $0.767^{*}$ & $0.464^{*}$ & $0.540^{*}$ \\
\hline $\begin{array}{l}\text { Anterior facial height } \\
\qquad(\mathrm{N}-\mathrm{Me})\end{array}$ & 0.062 & -0.235 & 0.122 & 0.254 & -0.081 & $0.819^{*}$ & $0.554^{*}$ & $0.416^{*}$ \\
\hline Pog-NB & 0.011 & -0.016 & 0.157 & 0.097 & 0.005 & 0.094 & 0.309 & 0.237 \\
\hline U1-SN & -0.289 & 0.155 & 0.289 & $0.441^{*}$ & 0.308 & -0.002 & 0.218 & $0.385^{*}$ \\
\hline IMPA & 0.024 & -0.012 & 0.173 & -0.022 & -0.096 & 0.147 & 0.184 & 0.211 \\
\hline
\end{tabular}

* statistical significance.

Table 6. Pearson's correlation between skeletal/dental changes and soft tissue changes in the extraction group

\begin{tabular}{|c|c|c|c|c|c|c|c|c|}
\hline $\begin{array}{l}\text { Changes } \\
\text { in parameters }\end{array}$ & $\begin{array}{l}\text { Nasolabial angle } \\
\text { (Col-Sn-UL) }\end{array}$ & $\begin{array}{l}\text { Upper lip to } \\
\text { E-plane }\end{array}$ & $\begin{array}{l}\text { Lower lip to } \\
\text { E-plane }\end{array}$ & $\begin{array}{l}\text { Upper lip } \\
\text { thickness }\end{array}$ & $\begin{array}{l}\text { Lower lip } \\
\text { thickness }\end{array}$ & $\begin{array}{l}\text { Upper lip } \\
\text { length }\end{array}$ & $\begin{array}{l}\text { Lower lip } \\
\text { length }\end{array}$ & $\begin{array}{l}\text { Mentolabial } \\
\text { sulcus }\end{array}$ \\
\hline $\begin{array}{l}\text { Posterior facial height } \\
\qquad \text { (S-Go) }\end{array}$ & -0.190 & -0.275 & 0.078 & $0.358^{*}$ & -0.730 & $0.895^{*}$ & $0.829^{*}$ & $0.346^{*}$ \\
\hline $\begin{array}{l}\text { Anterior facial height } \\
\qquad(\mathrm{N}-\mathrm{Me})\end{array}$ & -0.258 & -0.229 & 0.169 & $0.396^{*}$ & -0.032 & $0.922^{*}$ & $0.823^{*}$ & $0.411^{*}$ \\
\hline Pog-NB & -0.130 & -0.225 & $-0.347^{*}$ & 0.240 & 0.002 & 0.324 & $0.409^{*}$ & -0.050 \\
\hline U1-SN & -0.085 & -0.115 & 0.005 & 0.203 & 0.293 & 0.048 & 0.034 & 0.122 \\
\hline IMPA & -0.156 & -0.067 & 0.229 & 0.176 & 0.051 & $0.382^{*}$ & 0.197 & $0.369^{*}$ \\
\hline
\end{tabular}

* statistical significance.

\section{Discussion}

In general, dental and skeletal changes that occur during orthodontic treatment affect the soft tissue structures. Soft tissue changes do not completely coincide with hard tissue changes, and may be greater or lesser depending on the treatment plan. ${ }^{5,13-16}$ The purpose of the present study was to compare the treatment outcomes between the extraction and non-extraction orthodontic treatment groups.

\section{Soft tissue variables}

In both the extraction and non-extraction groups, lower lip to E-plane changed significantly. In the extraction group, the lower lip moved backward whereas in the nonextraction group, the lower lip moved forward. In a study by $\mathrm{Xu}$ et al., the lower lip moved backward in relation to E-plane in both extraction and non-extraction treatment, but this movement was greater in the extraction group. ${ }^{17}$ However, in extraction and non-extraction treatment, the lower lip moved backward and forward in relation to Sn-Pog line (the line passing through subnasale and the chin prominence), respectively. Thus, it seems that the change in the lower lip position was similar to that reported in the present study. The reason for the difference in the results regarding lower lip to E-plane can be attributed to the age range and malocclusion of the patients in the study by Xu et al. In their study, the mean age of patients was 12 years and a mixed group of patients with different malocclusion types was evaluated. ${ }^{17}$ Large changes in the nasal tip prominence can make E-line an unstable plane.
In addition, Momeni Danaei et al. examined the skeletal, dental and soft tissue changes following orthodontic treatment with or without tooth extraction in patients with class II division I malocclusion, and reported results similar to the results of the extraction group in the present study. ${ }^{15}$ However, in the non-extraction group, the lower lip was not significantly altered. This difference in the outcome is due to the difference in the malocclusion type (class I vs class II). In other words, in class II patients, both extraction and non-extraction treatment caused similar changes in the dental position. ${ }^{15}$ These results were similar to those of Bravo. ${ }^{18}$ Also, Bishara et al. reported the forward movement of the lower lip during non-extraction treatment. ${ }^{19}$

The comparison of the 2 groups revealed that changes in the position of the lower lip in the extraction group were significantly different from those in the non-extraction group, which is similar to the findings of $\mathrm{Xu}$ et al. ${ }^{17}$

Upper lip to E-plane experienced significant changes in the extraction group. It seems that the upper lip moved backward in the extraction group in our study. This finding is similar to that of Bravo. ${ }^{18}$

There was a significant difference between the groups in the position of the upper lip in relation to E-plane in our study. Our results are in contrast to those of Xu et al., which can be attributed to the treatment mechanics. ${ }^{17}$

In our study, nasolabial angle in the extraction group significantly increased, which suggests that the upper lip moved backward. This angle decreased very slightly in the non-extraction group after treatment, but the change was not statistically significant and may indicate that the upper lip position did not change significantly. 
This finding confirms the results regarding the position of the lips in relation to E-plane. It is also similar to the results of Talass et al., ${ }^{20}$ Lo et al. ${ }^{21}$ and Diels et al. ${ }^{22}$ Changes in this angle did not differ significantly between the 2 groups.

In our study, mentolabial sulcus increased significantly in the non-extraction group after treatment, but decreased significantly in the extraction group. This finding shows that mandibular incisor retroclination in the extraction group can retract the lower lip relative to the sagittal plane. Also, in the non-extraction group, as the mandibular incisors moved forward, the lower lip also moved toward the sagittal plane, causing a deeper mentolabial sulcus. ${ }^{1}$ These changes are in line with significant changes in IMPA, which were observed in both groups after treatment. The mentolabial sulcus changes also coincided with the lower lip to E-plane changes. In the non-extraction group, lower lip to E-plane significantly decreased after treatment, which indicated the protrusion of the lower lip. In the extraction group, the distance between the lower lip and E-plane significantly increased, which indicated the retrusion of the lower lip. The mentolabial sulcus changes were different between the 2 groups.

The lip thickness and length did not change in any group. The results of other studies in this regard have been controversial. ${ }^{5,9}$ Such contradictory findings indicate that multiple factors, other than orthodontic treatment, can affect the thickness of the lips, such as age, race and the lip structure.

No significant difference was observed in the interlabial gap in any group. Due to the fact that the patients in this study were in the borderline spectrum, none of them had a severe protrusion or retrusion of the lips. Hence, achieving this finding seems logical.

\section{Skeletal variables}

In the anteroposterior aspect, ANB and Wits appraisal did not change significantly in any group. Considering the fact that all patients had class I malocclusion before treatment, this finding appears to be logical.

In the extraction group, Pog-NB significantly increased after treatment. This finding suggests that in the extraction group, the chin seems to be more prominent in the profile view. This variable did not change significantly in the non-extraction group. Although point B is a skeletal point, a change in the position of the teeth can affect the position of this point. ${ }^{23}$ The retraction of the lower teeth could retract NB line and make Pog-NB larger in the extraction group.

In the vertical dimension, the posterior and anterior facial height did not change significantly in either group. The only significant change in the vertical dimension was in the mandibular plane and the cranial base angle (SN-GoMe) in the non-extraction group. The measurements showed that this angle increased slightly after treatment in the nonextraction group. However, the linear variables of the vertical facial height did not change significantly.

\section{Dental variables}

In the extraction group, U1-SN decreased significantly after treatment, indicating the retraction of the maxillary incisors. This finding is consistent with a significant decrease in upper lip to E-plane in the extraction group. This may indicate that with the retraction of the maxillary incisors, the upper lip also moved backward. The parameter U1-SN significantly increased in the non-extraction group, which caused the upper lip to move forward relative to the sagittal plane. It should be noted that upper lip to E-plane increased in the non-extraction group, but this increase was not large enough to cause a statistically significant change. It also shows that the upper lip changes did not fully coincide with dental changes.

Changes in the upper incisor inclination in the extraction group were significantly different from those in the non-extraction group. The parameter IMPA significantly increased in the non-extraction group, but significantly decreased in the extraction group. This finding is in agreement with the findings regarding lower lip to E-plane. Following the retraction of the incisors, the lower lip moved backward in the extraction group whereas in the nonextraction group, the lower lip moved forward due to the protraction of the incisors. The differences between the 2 treatment groups in the angle between the lower incisors and the mandibular plane were significant.

Interincisal angle increased significantly in the extraction group, which can be attributed to a significant reduction in $\mathrm{U} 1-\mathrm{SN}$ and IMPA in the extraction group. Interincisal angle decreased significantly in the non-extraction group, which explains the maxillary and mandibular incisor protrusion. There was a significant difference between the 2 groups in the interincisal angle variations.

\section{Correlation between the variables}

In the present study, the inclination of incisors did not show a strong correlation with the upper lip position. This angle did not have a significant correlation with nasolabial angle, either. In fact, the position of the upper lip was not significantly correlated with any of the evaluated variables. However, the position of the lower lip in relation to E-plane had a significant inverse correlation with Pog-NB in the extraction group. The variable Pog-NB did not have a positive correlation with lower lip to E-plane in the non-extraction group. The lower lip thickness did not have a significant correlation with any of the vertical or dental variables, but the upper lip thickness had a significant positive correlation with the angle of the maxillary incisors (U1-SN) in the non-extraction group as well as with the posterior and anterior facial height in the extraction group. Nasolabial angle did not correlate with any hard tissue variable. This finding is similar to that of Fitzgerald et al. ${ }^{24}$ 
Upper lip to E-plane did not have any correlation with the hard tissue variables. However, lower lip to E-plane had an inverse correlation with the chin position (Pog-NB) in the extraction group. As the chin moves forward, E-plane is also displaced forward, increasing the lower lip-to-E-plane distance.

The lower lip thickness did not have any significant correlation with the hard tissue variables. However, the upper lip thickness had a positive correlation with the upper incisor angulation (U1-SN) in the non-extraction group, and the posterior and anterior facial height in the extraction group. Shirvani et al. also revealed a correlation between the incisor angulation and the lip thickness. ${ }^{25}$ The lip structure and its initial thickness can affect this correlation. Oliver showed that in patients with thicker lips or in people who have lips with low traction, the lips follow hard tissue changes to a lesser degree in comparison with patients with thin lips or high-traction lips. ${ }^{26}$ A lack of a correlation between the upper lip thickness and the incisor angulation in the extraction group can be attributed to this fact. In addition, Momeni Danaei et al. revealed that, although lip retraction had a correlation with dental retraction, this correlation was not strong. ${ }^{15}$ According to Talass et al., the upper lip changes during orthodontic treatment are less predictable, and they can be attributed to the lip anatomy and the complex dynamics of the upper lip. ${ }^{20}$

In both the extraction and non-extraction groups, a significant positive correlation existed between the vertical facial changes and the upper and lower lip length. It is interesting to note that in both groups, the facial height changes had a stronger correlation with the upper lip than with the lower lip.

In the extraction group, the chin prominence had a positive correlation with the lower lip length. The lower lip length is measured as the distance between soft tissue menton and the most superior point of the lower lip. It seems logical that by increasing the chin prominence, the lower lip length would increase.

The lower incisor angulation had a significant correlation with the upper lip length in the extraction group. It seems that the retraction of the lower incisors decreases the traction of the upper lip, leading to lip shortening. ${ }^{25}$

There was a significant positive correlation between the facial height and mentolabial sulcus. Mentolabial sulcus had a positive correlation with the angle of the maxillary incisors in the non-extraction group and the angle of the mandibular incisors in the extraction group. This finding is similar to that of Bloom. ${ }^{27}$

\section{Conclusions}

According to the results of the present study, extraction and non-extraction orthodontic treatment has different effects on the soft tissue profile of borderline class I orthodontic patients. In general, skeletal changes were similar in the 2 groups. The greatest difference was in the lips and the upper and lower incisors. In the patients with the extraction treatment plan, the anterior teeth and the upper and lower lips were retracted relative to the sagittal plane, but in the patients with the non-extraction treatment plan, the anterior teeth and the lower lip moved forward relative to the sagittal plane.

Mentolabial sulcus also became shallower after treatment in the extraction group and deeper in the nonextraction group. Considering these findings along with the patients' facial features can be beneficial to the orthodontic treatment planning of borderline patients.

\section{ORCID iDs}

Sepideh Soheilifar (10) https://orcid.org/0000-0003-3515-1637 Sanaz Soheilifar (1) https://orcid.org/0000-0002-9890-5136 Hooman Ataei (1) https://orcid.org/0000-0002-5598-0259 Vahid Mollabashi (1) https://orcid.org/0000-0002-8450-7604 Payam Amini (1) https://orcid.org/0000-0001-8675-0045 Anahita Bakhshaei (1) https://orcid.org/0000-0003-4574-5797 Navid Naghdi (1) https://orcid.org/0000-0003-2991-2030

\section{References}

1. Amirabadi GE, Mirzaie M, Kushki SM, Olyaee P. Cephalometric evaluation of soft tissue changes after extraction of upper first premolars in class II div 1 patients. J Clin Exp Dent. 2014;6(5):e539-e545.

2. Maple JR, Vig KWL, Beck FM, Larsen PE, Shanker S. A comparison of providers' and consumers' perceptions of facial-profile attractiveness. Am J Orthod Dentofacial Orthop. 2005;128(6):690-696, quiz 801.

3. Soh J, Chew MT, Wong HB. A comparative assessment of the perception of Chinese facial profile esthetics. Am J Orthod Dentofacial Orthop. 2005;127(6):692-699.

4. Wilmot JJ, Barber HD, Chou DG, Vig KW. Associations between severity of dentofacial deformity and motivation for orthodonticorthognathic surgery treatment. Angle Orthod. 1993;63(4):283-288.

5. Verma SL, Sharma VP, Tandon P, Singh GP, Sachan K. Comparison of esthetic outcome after extraction or non-extraction orthodontic treatment in class II division 1 malocclusion patients. Contemp Clin Dent. 2013;4(2):206-212.

6. Kachiwala VA, Kalha AS, Machado G. Soft tissue changes associated with first premolar extractions in adult females. Aust Orthod J. 2009;25(1):24-29.

7. Leonardi R, Annunziata A, Licciardello V, Barbato E. Soft tissue changes following the extraction of premolars in nongrowing patients with bimaxillary protrusion: A systematic review. Angle Orthod. 2010;80(1):211-216.

8. Zarringhalam M, Arash V. Labial changes following extraction of first premolars for orthodontic treatment in patients with malocclusion class II Div I [in Persian]. J Mashhad Dent School. 2003;27(3,4):126-133.

9. Erdinc $A E$, Nanda RS, Dandajena TC. Profile changes of patients treated with and without premolar extractions. Am J Orthod Dentofacial Orthop. 2007;132(3):324-331.

10. Bishara SE, Jakobsen JR. Profile changes in patients treated with and without extractions: Assessments by lay people. Am J Orthod Dentofacial Orthop. 1997;112(6):639-644.

11. Hazar S, Akyalçin S, Boyacioğlu H. Soft tissue profile changes in Anatolian Turkish girls and boys following orthodontic treatment with and without extractions. Turk J Med Sci. 2004;34(3):171-178.

12. Lai J, Ghosh J, Nanda RS. Effects of orthodontic therapy on the facial profile in long and short vertical facial patterns. Am J Orthod Dentofacial Orthop. 2000;118(5):505-513.

13. Paquette $D E$, Beattie JR, Johnston LE Jr. A long-term comparison of nonextraction and premolar extraction edgewise therapy in "borderline" Class II patients. Am J Orthod Dentofacial Orthop. 1992;102(1):1-14. 
14. Basciftci FA, Usumez S. Effects of extraction and nonextraction treatment on class I and class II subjects. Angle Orthod. 2003;73(1):36-42.

15. Momeni Danaei S, Salehi P, Zareh A, Keshavarz M. Soft tissue and dentoskeletal changes in class II division 1 patients following extraction and non-extraction treatment [in Persian]. J Dent. 2005;6(1,2):128-138.

16. Xu TM, Liu Y, Huang W, Lin JX. Cephalometric comparison of softtissue morphology between extraction and non-extraction orthodontic treatment in borderline cases [in Chinese]. Beijing Da Xue Xue Bao Yi Xue Ban. 2004;36(6):650-654.

17. $\mathrm{Xu} T \mathrm{TM}$, Liu $\mathrm{Y}$, Yang MZ, Huang $\mathrm{W}$. Comparison of extraction versus nonextraction orthodontic treatment outcomes for borderline Chinese patients. Am J Orthod Dentofacial Orthop. 2006;129(5):672-677.

18. Bravo LA. Soft tissue facial profile changes after orthodontic treatment with four premolars extracted. Angle Orthod. 1994;64(1):31-42.

19. Bishara SE, Cummins DM, Jakobsen JR, Zaher AR. Dentofacial and soft tissue changes in Class II, division 1 cases treated with and without extractions. Am J Orthod Dentofacial Orthop. 1995;107(1):28-37.

20. Talass MF, Talass L, Baker RC. Soft-tissue profile changes resulting from retraction of maxillary incisors. Am J Orthod Dentofacial Orthop. 1987;91(5):385-394.

21. Lo FD, Hunter WS. Changes in nasolabial angle related to maxillary incisor retraction. Am J Orthod. 1982;82(5):384-391.

22. Diels RM, Kalra V, DeLoach N Jr., Powers M, Nelson SS. Changes in soft tissue profile of African-Americans following extraction treatment. Angle Orthod. 1995;65(4):285-292.

23. Al-Abdwani R, Moles DR, Noar JH. Changes of incisor inclination effects on point A and B. Angle Orthod. 2009;79(3):462-467.

24. Fitzgerald JP, Nanda RS, Currier GF. An evaluation of the nasolabial angle and the relative inclinations of the nose and upper lip. Am J Orthod Dentofacial Orthop. 1992;102(4):328-334.

25. Shirvani A, Sadeghian S, Abbasi S. Prediction of lip response to orthodontic treatment using a multivariable regression model. Dent Res J (Isfahan). 2016;13(1):38-45.

26. Oliver BM. The influence of lip thickness and strain on upper lip response to incisor retraction. Am J Orthod. 1982;82(2):141-149.

27. Bloom LA. Perioral profile changes in orthodontic treatment. Am J Orthod Dentofacial Orthop. 1961;47(5):371-379. 
